\title{
Research on the Application of Numerical Method in Control Theory
}

\author{
Yuanyuan Zhang \\ College of Science, China Three Gorges University, Yichang, China
}

Email address:

mathzhyy@163.com

\section{To cite this article:}

Yuanyuan Zhang. Research on the Application of Numerical Method in Control Theory. Applied and Computational Mathematics. Vol. 6, No. 4, 2017, pp. 185-188. doi: 10.11648/j.acm.20170604.14

Received: April 4, 2017; Accepted: July 3, 2017; Published: July 19, 2017

\begin{abstract}
Time optimal control problems of ordinary differential equations have been of great interest for decades due to their practical applications. There are mainly two ways to compute optimal times. The first one is the Switching Time Optimization method, where the switching time is taken as extra unknowns and the optimization problems is solved by nonlinear programming technique. The second one is based on the first order necessary condition for optimal control. In this paper, we extend the numerical method given in [1] for the computation of the optimal time for the time optimal control problems. In the end some examples are provided to show the efficiency of the numerical method.
\end{abstract}

Keywords: Numerical Method, Ordinary Differential Equation, Time Optimal Control Problems

\section{Introduction}

Time optimal control problems of ordinary differential equations have been of great interest for decades due to their practical applications [1-6]. There are a lot of literatures on this issue (see for instance [7-16]). For a time optimal control problem, how to compute the optimal time should be an important and interesting subject. To the best of our knowledge, there are mainly two ways to compute optimal times. The first one is the Switching Time Optimization method, where the switching time is taken as extra unknowns and the optimization problems is solved by nonlinear programming technique. The second one is based on the first order necessary condition for optimal control. In [1], Lu, Wang and Yan provided a different way to compute the optimal time. First, they obtained a necessary and sufficient condition for the optimal time. Then they applied a bisection method to solve the nonlinear equation.

In this paper, we extend the method given by Lu, Wang and Yan in [1] for the computation of the optimal time for the linear ordinary differential equation. The rest of the paper is organized as follows: Section 2 presents the numerical method for the computation of the optimal time for the time optimal control problems governed by a linear ordinary equation. Section 3 gives some examples to show the efficiency of the new method. Section 4 is the conclusion of this paper.

\section{Main Results}

Let $A$ and $B$ be two matrix in $R^{n \times n}$ and $R^{n \times m}$ respectively, where $n, m \in N$. Consider the following controlled ordinary differential equation:

$$
\left\{\begin{array}{c}
y^{\prime}(t)+A y(t)=B u(t) \\
y(0)=y_{0}
\end{array}\right.
$$

Here $y_{0} \in R^{n} \backslash\{0\}$ and $u$ is a control function in the following admissible set:

$$
\mu_{M}=\left\{v:[0,+\infty) \rightarrow R^{m},\|v(t)\|_{R^{M}} \leq M\right\},
$$

where $M$ is a positive constant. Throughout this paper, we use $(A, B)$ to denote the linear differential system given by equation (1). Clearly, when $y_{0}$ is given, for each $u \in$ $L^{\infty}\left(R^{+}, R^{m}\right),(1)$ has a unique solution which will be denoted by $y(t, u)$.

In this paper, we will extend the numerical method given in [1] for the computation of the optimal time for the following time optimal control problem:

$$
\left(P_{M}\right) \min \left\{T>0: y(t, u)=0, u \in \mu_{M}\right\} .
$$

In this problem, the number 


$$
t^{*}(M) \equiv \min \left\{T>0: y(t, u)=0, u \in \mu_{M}\right\}
$$

is called the optimal time; a control $u_{M}^{*} \in \mu_{M}$ is called an optimal control when $y\left(t^{*}(M) ; u_{M}^{*}\right)=0$ and $u^{*}(\cdot)=0$ over $\left(t^{*}(M), \infty\right)$; a control $u \in \mu_{M}$ is called an admissible control for Problem $\left(P_{M}\right)$, if there exists a time $T>0$ such that $y(T, u)=0$ and $u(\cdot)=0$ over $(T, \infty)$. At the same time, we introduce the following norm optimal control problem for each $T>0$ :

$$
(N P)_{T} M^{*}(T) \equiv \min \left\{\|u\|_{L^{\infty}\left(0, T ; R^{m}\right)}: y\left(T ; \chi_{[0, T)} u\right)=0\right\},
$$

where $y\left(T ; \chi_{[0, T)} u\right)$ satisfies equation (1) for $t \in(0, T)$.

In [1], Lu, Wang and Yan obtained the following results. We provide their proofs for the purpose that the readers can easily read the paper.

Lemma 1 Let $T, M>0$, then $T=t^{*}\left(M^{*}(T)\right)$ and $M=M^{*}\left(t^{*}(M)\right)$. Consequently,

(1) Problems $(N P)_{T}$ and $\left(P_{M^{*}(T)}\right)$ are equivalent, i.e., they have the same optimal control;

(2) Problems $\left(P_{M}\right)$ and $(N P)_{t^{*}(M)}$ are equivalent.

Proof. We first show that

$$
T=t^{*}\left(M^{*}(T)\right), \forall T \in(0,+\infty) .
$$

Indeed, let $u$ be an optimal control to $(N P)_{T}$. Then

$$
\|u\|_{L^{\infty}\left(0, T ; R^{m}\right)}=M^{*}(T) \text { and } y(T, u)=0 .
$$

By the optimality of $t^{*}\left(M^{*}(T)\right)$ to $\left(P_{M^{*}(T)}\right)$, we get that

$$
t^{*}\left(M^{*}(T)\right) \leq T \text {. }
$$

If $t^{*}\left(M^{*}(T)\right) \leq T$, then there would be a positive number

$M_{1}<M^{*}(T)$ such that $T=t^{*}\left(M_{1}\right)$. Let $u_{M_{1}}^{*}$ be the optimal control to $\left(P_{M_{1}}\right)$. Then we have that

$$
\left\|u_{M_{1}}^{*}\right\|_{L^{\infty}\left(0, t^{*}\left(M_{1}\right) ; R^{m}\right)}=M_{1}<M^{*}(T),
$$

and

$$
y\left(T ; u_{M_{1}}^{*}\right)=y\left(t^{*}\left(M_{1}\right) ; u_{M_{1}}^{*}\right)=0 .
$$

The latter indicates $\left\|u_{M_{1}}^{*}\right\|_{L^{\infty}\left(0, T ; R^{m}\right)} \geq M^{*}(T)$, which contradicts with (9). Hence (8) holds.

Next, by (8), we have that $t^{*}(M)=t^{*}\left(M^{*}\left(t^{*}(M)\right)\right)$. Since $t^{*}(\cdot)$ is strictly monotone, we get that

$$
M=M^{*}\left(t^{*}(M)\right), \forall M>0 .
$$

Finally, by (8) and (11) and by the definition of optimal controls to $\left(P_{M}\right)$ and $(N P)_{T}$, one can easily verify the desired equivalences.

Lemma 2 The function $M^{*}(\cdot)$ is strictly decreasing and continuous. In addition, it holds that $\lim _{T \rightarrow+\infty} M^{*}(T)=0$ and $\lim _{T \rightarrow 0^{+}} M^{*}(T)=+\infty$.

Proof. From Lemma 1, it follows that $M^{*}(\cdot)$ is the inverse function of $t^{*}(\cdot)$. This completes the proof.

A necessary and sufficient condition for the optimal time $t^{*}(M)$ will be given through using the above equivalence theorem. To this end, for each $T>0$, we introduce a function $J^{T}(\cdot): R^{n} \rightarrow R$ as follows:

$$
J^{T}(\mu)=\frac{1}{2}\left(\int_{0}^{T}\|E(t, T) \mu\| d t\right)^{2}+<e^{-A T} y_{0}, \mu>0,
$$

where $E(t, T)=B^{T} e^{A^{T}(t-T)}$.

Lemma 3 Let $T>0$. Then $\operatorname{Min}_{\mu \in R^{n J}} J^{T}(\mu)$ has at least one minimizer. Furthermore,

$$
M^{*}(T)=\int_{0}^{T}\left\|E(t, T) \mu_{T}^{*}\right\| d t .
$$

Lemma 4 Let $u_{M}^{*}$ be an optimal control for $\left(P_{M}\right)$, Then $\left\|u_{M}^{*}\right\|=M$ for a.e. $t \in\left(0, t^{*}(M)\right)$.

Lemma 5 Let $M>0$. Then, $\widetilde{T}$ is the optimal time to Problem $\left(P_{M}\right)$, i.e. $\tilde{T}=t^{*}(M)$, if and only if for each minimizer $\mu_{T}^{*}$ of $J^{T}(\cdot), \widetilde{T}>0$ solves the following nonlinear equation:

$$
M=\int_{0}^{T}\left\|E(t, T) \mu_{T}^{*}\right\| d t, T>0 .
$$

Moreover, when $\mu_{T}^{*}$ is a minimizer of $J^{T}(\cdot)$, the above equation has a unique solution.

Proof. Clearly, $t^{*}(M)>0$. From $(11)$ and Lemma 3 , it follows that for each minimizer $\mu_{T}^{*}$ of $J^{T}(\cdot), t^{*}(M)$ solves equation (14).

Conversely, suppose that $\widetilde{T}>0$ is a solution to equation (14) for some minimizer $\mu_{T}^{*}$ of $J^{T}(\cdot)$. Then, by (13) with $T=\tilde{T}$, it holds that $M=M^{*}(\widetilde{T})$. Since $M^{*}\left(t^{*}(M)\right)=\mathrm{M}$, $M^{*}(\widetilde{T})=M^{*}\left(t^{*}(M)\right)$. This, along with the strict monotonicity of $M^{*}(\cdot)$, indicates that $\tilde{T}=t^{*}(M)$.

Finally, we show the uniqueness. Let $\mu_{T}^{*}$ be a minimizer of $J^{T}(\cdot)$. By Lemma 3, Equation (14) is equivalent to the equation:

$$
M=M^{*}(T), T>0 .
$$

By Lemma 2, the above equation has a unique solution, so is Equation (14). This completes the proof.

Based on the lemmas given above, we will build up the following theorem for the computation of the optimal time for the linear ordinary differential equation:

$$
\left\{\begin{array}{c}
y^{\prime}(t)+a y(t)=b u(t) \\
y(0)=y_{0}
\end{array}\right.
$$

where $y_{0} \neq 0, a>0$ and $b \neq 0$.

Theorem Let $t^{*}(M)$ be an optimal control for the linear ordinary differential equation (16), then

$$
t^{*}(M)=-\frac{1}{a} \ln \frac{M|b|}{M|b|+a\left|y_{0}\right|}
$$

Proof. For the system (16), the function $J^{t^{*}(M)}: R \rightarrow R$ now reads:

$$
J^{t^{*}(M)}(\mu)=\frac{1}{2}\left(\int_{0}^{t^{*}(M)}\|E(t, T) \mu\| d t\right)^{2}+<e^{-A T} y_{0}, \mu>0
$$

i.e., 


$$
J^{t^{*}(M)}(r)=\frac{1}{2}\left(\int_{0}^{t^{*}(M)}\left|b e^{a\left(t-t^{*}(M)\right)} r\right| d t\right)^{2}+e^{-a t^{*}(M)} y_{0} r .
$$

Case 1. When $a>0$, it holds that

$$
J^{t^{*}(M)}(r)=\frac{b^{2}}{2 a^{2}}\left(1-e^{-a t^{*}(M)}\right)^{2} r^{2}+e^{-a t^{*}(M)} y_{0} r .
$$

$$
\text { By solving }\left(U^{t^{*}(M)}(r)\right)^{\prime}=0 \text {, }
$$

we can easily obtain the following unique minimizer of $J^{t^{*}(M)}(\cdot)$ :

$$
r^{*}=\frac{a^{2} e^{-a t^{*}(M)} y_{0}}{b^{2}\left(1-e^{-a t^{*}(M)}\right)^{2}}
$$

Then by Lemma 4, we have

$$
M=\int_{0}^{t^{*}(M)}\left|b e^{a\left(t-t^{*}(M)\right)} r^{*}\right| d t .
$$

Then it is obvious that

$$
M=\frac{a\left|y_{0}\right|}{|b|}\left(\frac{1}{1-e^{-a t^{*}(M)}}-1\right) .
$$

Then it is easy to obtain

$$
t^{*}(M)=-\frac{1}{a} \ln \frac{M|b|}{M|b|+a\left|y_{0}\right|} .
$$

Case 2. When $a=0$, by the same method, we can get

$$
t^{*}(M)=\frac{\left|y_{0}\right|}{M|b|} \text {. }
$$

This completes the proof.

\section{Applications}

In this section we provide several examples to show the efficiency of the above-mentioned method.

Example 1. Let

$$
\left\{\begin{array}{c}
\mathrm{y}^{\prime}(\mathrm{t})+2 \mathrm{y}(\mathrm{t})=2 \mathrm{u}(\mathrm{t}) \\
\mathrm{y}(0)=1
\end{array}\right.
$$

and $M=1$, we aim to compute $t^{*}(1)$.

Solution. In this example, $a=2>0, b=2>0, y_{0}=$ $1, M=1$, according to case 1 in the theorem in section 2 , it is easy to obtain

$$
t^{*}(1)=-\frac{1}{a} \ln \frac{M|b|}{M|b|+a\left|y_{0}\right|}=\frac{\ln 2}{2} .
$$

Example 2. Let

$$
\left\{\begin{array}{c}
y^{\prime}(t)+y(t)=u(t) \\
y(0)=1
\end{array}\right.
$$

and $M=2$, we aim to compute $t^{*}(2)$.

Solution. In this example, $a=-1<0, b=1>0, y_{0}=$ $1, M=2$, according to case 1 in the theorem in section 2 , it is easy to obtain

$$
t^{*}(2)=-\frac{1}{a} \ln \frac{M|b|}{M|b|+a\left|y_{0}\right|}=\ln 2 .
$$

Example 3. Let

$$
\left\{\begin{array}{c}
y^{\prime}(t)+y(t)=-u(t), \\
y(0)=1,
\end{array}\right.
$$

and $M=3$, we aim to compute $t^{*}(3)$.

Solution. In this example, $a=1, b=-1<0, y_{0}=$ $1, M=3$, according to case 1 in the theorem in section 2 , it is easy to obtain

$$
t^{*}(3)=-\frac{1}{a} \ln \frac{M|b|}{M|b|+a\left|y_{0}\right|}=2 \ln 2-\ln 3 .
$$

Example 4. Let

$$
\left\{\begin{array}{c}
y^{\prime}(t)=2 u(t) \\
y(0)=1
\end{array}\right.
$$

and $M=1$, we aim to compute $t^{*}(1)$.

Solution. In this example, $a=0, b=2>0, y_{0}=$ $1, M=1$, according to the theorem in section 2 , it is easy to obtain

$$
t^{*}(1)=\frac{\left|y_{0}\right|}{M|b|}=\frac{1}{2} .
$$

Example 5. Let

$$
\left\{\begin{array}{c}
y^{\prime}(t)=-2 u(t) \\
y(0)=1
\end{array}\right.
$$

and $M=2$, we aim to compute $t^{*}(2)$.

Solution. In this example, $a=0, b=-2<0, y_{0}=$ $1, M=2$, according to the theorem in section 2 , it is easy to obtain

$$
t^{*}(2)=\frac{\left|y_{0}\right|}{M|b|}=\frac{1}{4} .
$$

Example 6. Let

$$
\left\{\begin{array}{c}
y^{\prime}(t)=-u(t) \\
y(0)=-1
\end{array}\right.
$$

and $M=3$, we aim to compute $t^{*}(3)$.

Solution. In this example, $a=0, b=-1<0, y_{0}=$ $-1, M=3$, according to the theorem in section 2 , it is easy to obtain

$$
t^{*}(3)=\frac{\left|y_{0}\right|}{M|b|}=\frac{1}{3} .
$$

\section{Conclusion}

This paper extends the numerical method given in [1] for the computation of the optimal time for the time optimal control problems. Section 2 presents the numerical method for the time optimal control problems governed by a linear ordinary equation. Section 3 gives some examples to show the efficiency of the new method. Section 4 is the conclusion of this paper. This method can also be used in other linear ordinary differential systems. 


\section{References}

[1] X. Lu, L. Wang and Q. Yan, "Computation of Time Optimal Control Problems governed by Linear Ordinary Differential Equations", Journal of Scientific Computing, 2017, pp. 1-25.

[2] V. Barbu, Analysis and Control of Nonlinear Infinite Dimensional Systems, Academic Press, Boston, 1993, pp. 74-98.

[3] L. C. Evans, An Introduction to Mathematical Optimal Control Theory, Lecture Notes, Department of Mathematics, University of California, Berkeley, 2008, pp. 92-101.

[4] H. O. Fattorini, Infinite Dimensional Linear Control Systems, the Time Optimal and Norm Optimal Control Problems, North-Holland Mathematics Studies 201, Elsevier, 2005, pp.48-67.

[5] K. Ito and K. Kunisch, "Semismooth newton methods for time-optimal control for a class of ODES", SIAM J. Control Optim., vol. 48, 2010, pp. 3997-4013.

[6] C. Y. Kaya and J. L. Noakes, "Computations and time-optimal controls", Optimal Control Applications and Methods, vol.17, 1996, pp.171-185.

[7] C. Y. Kaya and J. L. Noakes, "Computational methods for time-optimal switching controls", J. Optim. Theory Appl., vol. 117, 2003, pp. 69-92.

[8] J. P. Lasalle, The Time Optimal Control Problem, Contributions to the Theory of Nonlinear Oscillations, Princeton University Press, Princeton, 1960, 1-24.

[9] X. Li and J. Yong, Optimal Control Theory for Infinite Dimensional Systems, Birkhauser, Boston, 1995, pp. 127-135.

[10] P. Lin and G. Wang, "Blowup time optimal control for ordinary differential equations", SIAM J. Control Optim., vol. 49, 2011, pp. 73-105.

[11] E. Meier and A. E. Bryson, Efficient algorithms for time-optimal rigid spacecraft reorientation problem, Journal of Guidance, Control, and Dynamics, vol. 13, 1990, pp. 859-866.

[12] T. Li and B. Rao. "A note on the exact synchronization by groups for a coupled system of wave equations", Mathematical Methods in the Applied Sciences, vol. 38, 2015, pp. 241-246.

[13] L. Hu, F. Ji and K. Wang. "Exact boundary controllability and exact boundary observability for a coupled system of quasilinear wave equations", Chinese Annals of Mathematics, vol.34 (4), 2013, pp. 479-490.

[14] B. Z. Guo, D. H. Yang and L. Zhang. "On optimal location of diffusion and related optimal control for null controllable heat equation", Journal of Mathematical Analysis and Applications, vol. 433 (2), 2016, pp. 1333-1349.

[15] M. Chen. "Bang bang property for time optimal control of the korteweg de vries burgers equation", appl. math. opt., vol. 2, 2016, pp. 1-16.

[16] L. Wang and Q. Yan. "Time Optimal Controls of Semilinear Heat Equation with Switching Control", Journal of Theory and Applications, vol. 165 (1), 2015: 263-278. 